\title{
Assessment of Radiated Fan Noise Prediction Capabilities using Static Engine Test Data
}

\author{
Douglas M. Nark* \\ NASA Langley Research Center, Hampton, VA 23681-2199, U.S.A
}

\begin{abstract}
This paper describes further assessment of the CDUCT-LaRC code via comparison with static engine test data. In an effort to improve confidence in the use of CDUCT-LaRC for liner optimization studies addressing realistic three-dimensional geometries, inlet radiated fan noise predictions were performed at $54 \%$ and $87 \%$ engine speed settings. Predictions were then compared with far-field measurements to assess the approach and implementation. The particular configurations were chosen to exercise the three-dimensional capability of CDUCT-LaRC and it's applicability to realistic configurations and conditions. At the 54\% engine speed setting, the predictions capture the general directivity and acoustic treatment effects quite well. Comparisons of the predicted and measured directivity at the $87 \%$ power setting were more problematic. This was likely due in part to the difficulties in source specification and possibly the nonlinear nature of buzz-saw tones at this engine operating condition. Overall, the approach captured the basic trends and provided a conservative estimate of liner effects from which relative performance metrics could be inferred.
\end{abstract}

\section{Introduction}

Increasing air traffic and more stringent aircraft noise regulations continue to expand requirements on aircraft noise prediction capabilities for conventional and unconventional aircraft configurations. Accurate aircraft engine noise prediction is an important component of overall aircraft system noise tools and the ability to predict fan noise within complex three-dimensional aircraft nacelle geometries is a valuable tool in studying low-noise designs. Recent years have seen the development of aeroacoustic propagation codes using various levels of approximation to obtain such a capability! 1 For instance, the propagation module of the NASA Langley developed CDUCT-LaRC code utilizes a parabolic approximation to the convected Helmholtz equation formulated by Dougherty ${ }^{2 / 3}$ This approach affords very efficient propagation calculations, thus allowing solutions for complex three-dimensional geometries to be handled with relatively low computational costs. However, this efficiency can come at the expense of reduced fidelity as the actual propagation direction may diverge from the preferred direction of the parabolic approximation. Additionally, loss of fidelity may occur when reflection and/or scattering of acoustic waves become important. Nevertheless, if appropriate care is taken to account for the assumptions of the parabolic approximation, CDUCT-LaRC provides an efficient framework in which to perform fully three-dimensional aeroacoustic computations. As higher fidelity three-dimensional codes become available, it would seem appropriate to incorporate these into a framework similar to CDUCT-LaRC and use them in a complementary fashion. For example, an optimal design could be achieved using the current CDUCT-LaRC propagation module in conjunction with higher fidelity methodologies currently under development. Following this paradigm, CDUCT-LaRC could be used to efficiently identify preliminary designs. More accurate and computational intensive methods could then be used to further refine the results. Additionally, the various propagation codes could utilize the existing capabilities of the CDUCT-LaRC acoustic radiation module to predict radiated sound power.

With these ideas in mind, it is desirable to further identify a "working envelope" within which to use the various approaches for realistic configurations under realistic operating conditions. This study complements earlier assessments ${ }^{14}[5]$ of CDUCT-LaRC by using static engine test data. The data was obtained under the NASA/Honeywell Engine Validation of Noise and Emissions Reduction Technology (EVNERT) Contract using the TECH977 technology demonstrator engine ${ }^{[6}$ Specifically, the CDUCT-LaRC code with an assumed source model was used to predict inlet radiated fan noise for two engine operating conditions and several nacelle liner configurations. Predictions were then compared with far-field measurements. The nacelle configurations included non-axisymmetric acoustic liners and

${ }^{*}$ Research Scientist, Structural Acoustics Branch, Research \& Technology Directorate, Senior Member AIAA 
therefore all CDUCT-LaRC computations were fully three dimensional. This is important given the desire to improve confidence in the use of CDUCT-LaRC for liner optimization studies addressing realistic geometries.

The remainder of the paper is organized as follows. Test configurations and experimental measurement facilities are first presented in Section III This is followed by a discussion of the computational approach in Section III Comparison of the predictions with measurements at the blade passage frequency (BPF) are presented in section IV] Finally, concluding remarks regarding some of the more significant results and further areas of interest are presented in Section $\mathrm{V}$

\section{Test Configurations and Acoustic Measurements}

As described in the EVNERT Phase I report, ${ }^{[6}$ testing on a full scale Honeywell TECH977 technology demonstrator engine was performed at Honeywell's San Tan test site located southeast of Phoenix, Arizona. Far-field acoustic measurements were made to characterize engine noise directivity, as well as acoustic treatment effects. As seen in figure 1a, the engine was mounted on the test stand such that the engine centerline is 10 feet $(3.05 \mathrm{~m})$ above the ground. The far-field measurement system (see figure $1 \mathrm{~b}$ ) consisted of 32 microphones distributed along a $160^{\circ}$ arc 100 feet $(30.48 \mathrm{~m})$ from the engine inlet. Only the first 18 microphone locations, extending 5 to 90 degrees from the engine inlet, were used for comparison with predictions.

Four different inlet configurations were tested. As presented in figure 2, these include a hardwall, a seamless single degree of freedom (SDOF) linear liner, and two splice configurations. The latter allowed examination of liner discontinuities. Predictions were performed for all four configurations to include fully three-dimensional nacelles. The length of the SDOF liner was $L=12.8 \mathrm{in} .(0.32 \mathrm{~m})$ and the inlet diameter near the axial midpoint of the liner was taken to be $D=33.4 \mathrm{in}$. $(0.85 \mathrm{~m})$. The hardwall and splice configurations were obtained by applying aluminum tape to the seamless liner. Testing was performed at a number of corrected engine speed settings, (N1C). In this study, BPF results for a low power (54\% corrected engine speed) representative of an approach condition and a high power (87\% corrected engine speed) representative of a takeoff condition were considered.

\section{Prediction Code Description}

Detailed discussions of the CDUCT-LaRC code have been presented previously 117 However, a brief description is useful for outlining the prediction process. The CDUCT-LaRC code calculates the propagation of a given acoustic source ahead of the fan face or aft of the exhaust guide vanes in the inlet or exhaust ducts, respectively. Subsequent to the internal duct propagation calculations, the code has the capability of computing the noise radiation outside the duct. In addition to the ducts being acoustically lined in specified areas, other issues that make the propagation and radiation analysis complex include:

1. Variable area circular or annular sections within the duct, resulting in non-uniform mean flow.

2. Bifurcations within the exhaust duct resulting in one or two C-shaped regions.

3. Non-uniform (i.e. circumferentially and axially segmented) acoustic lining on the duct wall.

The CDUCT-LaRC code is composed of five distinct modules: 1) input and output specification, 2) computational fluid dynamics (CFD) and acoustic grid generation, 3) background flow calculation, 4) duct acoustic propagation, and 5) duct acoustic radiation. All of the modules that currently make up the CDUCT-LaRC framework are utilized in this study. However, this discussion will focus on the propagation and radiation modules, as they are most pertinent to this investigation.

The duct propagation module is based on the CDUCT code developed by Dougherty $\sqrt{2[3}$ and extended by Lan .8 This utilizes a parabolic approximation to the convected Helmholtz equation (based on a potential mean flow) and offers a computationally efficient model that accounts for the complexities listed above. The CDUCT-LaRC code has been extended ${ }^{7}$ to allow a user to automatically perform multi-block propagation calculations. The grid connectivity is determined and data is transferred from upstream to downstream (in the acoustic propagation direction) blocks without user intervention. Results of the propagation module include the acoustic potential or pressure within the duct, which may be utilized by the radiation module for acoustic radiation calculations.

The duct radiation model is based on the Ffowcs Williams-Hawkings (FW-H) equation with a penetrable data surface ${ }^{9}$ Use of this equation for many acoustic problems has shown it to generally provide better results than the Kirchhoff formula for moving surfaces. Based on the background flow conditions and propagation solution, this 
module calculates the radiated acoustic pressure at various observer locations. Currently, the data surface is taken to be the nacelle inlet or exhaust plane for inlet or aft-fan cases, respectively.

Before proceeding to a discussion of the results, a few aspects of the CDUCT-LaRC implementation for these configurations are addressed. First, the inlet computational grid and an example internal propagation calculation are shown in figure 3 . This flowpath geometry follows that used by Schuster $e t$. al. in a previous seamless liner optimization effort for the same TECH977 engine $\frac{10}{}$ As mentioned previously, predictions were performed for all hardwall and lined configurations to exercise the three-dimensional capability of CDUCT-LaRC. For this example calculation, and subsequent results presented, the internal computational domain consisted of $(65 \times 181 \times 97)$ points in the radial, circumferential, and axial directions, respectively. This grid resolution was denser than that required for the hardwall and seamless liner configurations. However, it was specified in order to resolve the spliced configurations. This grid point count translated to six and four points per splice circumferentially for the two- and three-splice configurations, respectively. Predictions were also performed by doubling the resolution in the circumferential direction to 361 points, but minimal differences were seen in the radiated noise field. Therefore, the $(65 \times 181 \times 97)$ grid resolution was maintained and all predictions were performed as three-dimensional simulations due to the efficient nature of the propagation calculations. At this grid resolution, a single (one frequency) three-dimensional propagation and acoustic radiation prediction took approximately six minutes on a single workstation $2.0 \mathrm{GHz} \mathrm{CPU}$. The acoustic source is specified at the indicated plane upstream of the fan (see figure 3). The propagation module then employs the parabolic approximation to march the solution from the source to the inlet highlight (right to left in figure 3). The inlet highlight surface and predicted quantities are then used as the FW-H permeable source surface for subsequent radiation calculations. This prediction process required specification of the mean flow Mach number and acoustic source. Although non-uniform flows may be considered within CDUCT-LaRC, the mean flow for the current study was assumed to obey a Mach-Area relation. The Mach number profiles were the result of iterative calculations in which the source plane Mach number was adjusted to approximate Mach number values used in the aforementioned seamless liner optimization effort ${ }^{10}$ As presented in figure 4 this translated to source plane Mach number values of $M=0.38$ and $M=0.60$ for $54 \%$ and $87 \%$ N1C, respectively. It should be noted that these Mach number profile approximations affect source and in-duct acoustic content and are a source of uncertainty in the prediction inputs.

In addition to the mean flow Mach number, the acoustic source required by the propagation module was specified at the source plane indicated in figure 3. While CDUCT-LaRC can accept arbitrary source specification (i.e., it is not a modal code), it is often convenient to specify the acoustic source distribution in terms of duct modes. For situations in which the source pressure is available, this greatly simplifies the conversion to the required acoustic potential. However, when source information is not available, an assumption on the source description must be made. This is generally the case, and it was therefore of interest to investigate the acceptability of using random modal amplitude and phase input. The approach taken followed that described by Zlavog and Eversman ${ }^{1112}$ in a series of statistical studies into the effects of randomized modal source power and/or phase on attenuation in lined ducts. In this study, the source modal powers (and hence, amplitudes) and modal phases were allowed to vary randomly and independently. Thus, with equal probability of occurrence, the amplitude and phase for each cut-on source mode take any value in the intervals from 0 to 1 and 0 to $2 \pi$, respectively. This uncertainty in source specification will undoubtedly result in uncertainty in the predicted sound levels.

Finally, for the configurations involving acoustic treatment, an impedance boundary condition representing the locally reacting SDOF liner was required. Slightly differing values for the acoustic liner impedance are found in the EVNERT repor ${ }^{6}$ and optimization study of Schuster. ${ }^{10}$ However, the latter included plots of acoustic resistance and reactance at both engine speed settings of interest. From these plots, normalized acoustic impedance values of $\zeta_{54 \%}=(1,-1)$ and $\zeta_{87 \%}=(2,0)$ were assumed for the $54 \%$ and $87 \%$ engine speed settings, respectively. Similar to the previous comments on mean flow and acoustic source specification, uncertainty in the specified impedance values lead to uncertainty in the predicted results.

\section{Results and Discussion}

Fan noise predictions at the blade passage frequency (BPF) are compared with measured data along an arc 100 feet $(30.48 \mathrm{~m})$ from the engine inlet. Observer locations extend from $5^{\circ}$ to $90^{\circ}$ from the inlet in $5^{\circ}$ increments consistent with the EVNERT test campaign. Radiation angles greater than $90^{\circ}$ were not included in the predictions due to the nature of the FW-H source surface. Here, the amplitude and phase of each source mode were randomly selected over a number of trials using a uniform distribution. Note that for a specific engine speed setting, the same random seed was used for each configuration. This provided a consistent source description across the configurations from which acoustic treatment effects could be studied. As discussed by Zlavog and Eversman, ${ }^{11,12}$ for the case of all propagating 
circumferential and radial acoustic modes with random modal power and phase, transmitted power appears to be normally distributed. In their statistical studies, this also produced statistical distributions with the least standard deviation, suggesting the least variable estimate for realized attenuation.

Following this reasoning, the acoustic radiation at the aforementioned observer locations was taken to be normally distributed. Therefore, Student's t-distribution was used to statistically analyze the predicted results. For each configuration and power setting, 32 simulations were performed to produce a sample population from which statistical information could be inferred. Following the discussion in Coleman and Steele, $\frac{13}{13}$ the sample mean, $\bar{X}$, is computed as

$$
\bar{X}=\frac{1}{N} \sum_{i=1}^{N} X_{i}
$$

where $N$ is the number (32 in this case) of individual samples $X_{i}$. The sample standard deviation, $S_{X}$, is defined by

$$
S_{X}=\left[\frac{1}{N-1} \sum_{i=1}^{N}\left(X_{i}-\bar{X}\right)^{2}\right]^{1 / 2}
$$

and the sample standard deviation of the mean, $S_{\bar{X}}$, is given by

$$
S_{\bar{X}}=\frac{S_{X}}{\sqrt{N}} .
$$

Thus, the $95 \%$ confidence limit for a sample of $N$ measurements of $X$ taken from a Gaussian distribution may be defined as the random uncertainty, $P$, where

$$
P_{\bar{X}}=t S_{\bar{X}}=\frac{t S_{X}}{\sqrt{N}}
$$

and $t$ is determined by the t-distribution ( $t=2.0395$ for this case with $N=32$ and $95 \%$ probability). Considering sample populations drawn from a Gaussian parent distribution, the $\pm P$ interval provides the interval within which the population mean, $\mu$, is expected to lie with $95 \%$ confidence. The expression for this $95 \%$ confidence interval may be written

$$
\operatorname{Prob}\left(\bar{X}-P_{\bar{X}} \leq \mu \leq \bar{X}+P_{\bar{X}}\right)=0.95 .
$$

Thus, in addition to providing source information when specific modal source content is not available, the approach provides confidence intervals to aid in prediction assessment.

Application of the aforementioned analysis to the CDUCT-LaRC predictions for the 54\% engine speed leads to the comparisons provided in figures 5 6 . In figure 5, the predicted and measured directivity patterns for the hardwall configuration are presented. The amplitude of the predicted levels are relative to an arbitrary acoustic source strength. Thus, in order to compare with the measured directivity pattern, the highest predicted SPL was set equal to the highest measured SPL. Note that these maximum levels do not occur at the same directivity angle. Thus, this adjustment did not lead to a match of the predicted and measured levels at a specific directivity angle. The $95 \%$ confidence interval for the predicted quantities is denoted by error bars. Uncertainty information was not available for the measurements, hence these quantities do not include error bars. In general, the shape of the directivity patterns match fairly well, with discrepancies at the shallow directivity angles. In addition to the hardwall directivity patterns, the differences in radiated SPL between the hardwall and seamless liner configuration are provided. This quantity is indicative of the liner effectiveness and is independent of the assumed acoustic source strength. Thus, no adjustment of levels was necessary in presenting the difference in radiated sound between the hardwall and treated configurations. With the exception of a few observer angles, the predicted $95 \%$ confidence interval encompasses the measured values. As seen in both the measured and predicted quantities, the seamless liner was most effective at extreme lower and upper radiation angles. Similar comparisons are made for the two- and three-splice lined configurations in figure 6 As with the seamless configuration, the liner effects are fairly well captured with discrepancies at some shallow angles. It can also be seen that rigid splice effects are not pronounced at this low speed setting. Overall comparisons for all configurations at the 54\% engine speed setting are promising.

Following the low speed comparisons, results for the $87 \%$ engine speed setting are provided in figures 778 , Beginning with the hardwall directivity patterns in figure 7 , it can be seen that agreement between measured and predicted levels are not as good as was observed at the lower power setting. As in the previous case, the highest predicted SPL was set equal to the highest measured SPL for the hardwall directivity. At this high engine speed setting, it appears that the modes radiating at higher directivity angles were not sufficiently represented in the prediction. These may 
include both higher order circumferential and radial modes. Results for the two- and three-splice configurations further illustrate this point as reasonable comparisons are obtained at shallow angles with under-prediction at the higher directivity angles. Additionally, rigid splice effects in the radiated predictions are not as apparent as those seen in the measurements. This may be due to the rich modal content already present in the specified source and may be less evident when analyzing the results statistically (i.e., focusing on mean quantities). However, it should be noted that internal insertion loss predictions (generated by the internal duct propagation module) were consistent with splice effect predictions presented in the EVNERT report. Therefore, comparing the radiated quantities in only one plane (containing the microphone arc) may also be a concern.

Further details on the conditions at this engine speed setting provide some insight into the difficulties at the higher engine speed setting. First, with 22 fan blades and 53 vanes, circumferential modes resulting from Tyler-Sofrin interaction are not present at BPF. However, as discussed in the EVNERT report, ${ }^{6}$ at this engine speed the fan tip speed is supersonic. This leads to buzz-saw tones for which the source modal content is dominated by a single circumferential mode. The order of the circumferential mode is the same as the engine order. Therefore, for the BPF case considered, the $m=22$ circumferential mode should receive increased weighting (since, again, the fan has 22 blades). This is further illustrated in the work by Schuster $[10$ in which source modal decomposition shows the $m=21$ through $m=24$ circumferential modes to be dominant. The presence of the additional circumferential modes may be a result of Tyler-Sofrin type interaction ${ }^{\text {a }}$ between the $m=22$ mode and the single probe mounted in the nacelle (as seen in the figure 2). However, measured hardwall levels at shallow directivity angles clearly show the presence of well cut-on modes that radiate in these directions. Thus, difficulties in identifying the proper source modal content, in addition to possible nonlinear propagation associated with the buzz-saw tones, make the predictions at this engine speed setting more difficult.

In an effort to further investigate the effects of source modal content for the $87 \%$ engine speed setting, alternative combinations of circumferential mode orders were investigated. All cut-on radial modes were still included with random amplitude and phase. However, subsets of the initial full complement of all cut-on circumferential modes were specified. As an example, the results for the case in which only the $m=0$ and $m=21$ through $m=24$ circumferential modes (with all cut-on radial modes) were included in the source description are shown in figures 90 . In this case, it appears that the shape of the directivity pattern at higher angles is fairly well captured in figure 10 . However, it is clear that too much weighting is given to the $m=0$ mode, as the shallow angles are clearly over-predicted.

A number of additional source mode combinations were considered for the higher speed setting with marginal improvement in comparison between measured and predicted liner effectiveness. Additionally, predicted rigid splice effects were no more pronounced with the alternative source specifications. Thus, for the purpose of efficiently identifying preliminary low-noise concepts in a coupled design cycle, the initial source model appears appropriate. For this randomized source model, with all cut-on circumferential and radial modes included, predictions captured the general directivity and acoustic treatment effects quite well at the lower engine speed. The higher engine speed comparisons were more problematic, possibly due to the difficulties in source specification and the nonlinear nature of the buzz-saw tones. However, the general trends appear to be captured and a conservative estimate obtained from which relative performance metrics may be inferred.

\section{Concluding Remarks}

The current study aimed to further identify a "working envelope" within which the CDUCT-LaRC code with a randomized source model may be used to efficiently and accurately predict fan noise for realistic configurations under realistic operating conditions. Specifically, a further assessment $1 / 4 / 14$ of the CDUCT-LaRC code was carried out via comparison with TECH977 static engine test data taken under the NASA/Honeywell EVNERT Contract. It is recognized that discrepancies may result from uncertainty in specifying quantities such as the mean flow Mach number, acoustic source content, and acoustic liner impedance. However, examination of the results suggest the following:

- When source content is not available in a convenient form, specifying random power and phase to each cut-on mode can produce a reasonable source model. This is particularly true for cases in which many modes are present. It is also possible to infer statistical information from this source model.

- Differences in predicted directivity patterns for the various splice configurations were not clearly evident, particularly at $87 \%$ N1C. This may be due to the rich modal content already present in the specified source. Additionally, these effects may be less evident when viewing mean values. Finally, although not presented in this work, insertion loss predictions were consistent with calculations presented in the EVNERT report.

${ }^{\mathrm{a}}$ For example, $22 \pm 1$ leads to $m=21,23$ 
- At the 54\% engine speed setting, the predictions of the BPF directivity pattern and acoustic treatment effects are in generally good agreement with measurements.

- At the $87 \%$ power setting, the agreement between predicted and measured directivity was not as good as the lower setting. This was likely due in part to the difficulties in source specification and possibly the nonlinear nature of the buzz-saw tones. However, the approach appears to capture the basic trends and provide a conservative estimate of acoustic liner performance.

- Based on these results, a preliminary liner optimization study will be initiated using the randomized source with all cut-on circumferential and radial modes.

\section{Acknowledgments}

The author wishes to thank Mr. Don Weir for his assistance with the TECH977 geometry and guidance concerning the EVNERT test. This research was funded by the Subsonic Fixed Wing Project of NASA's Fundamental Aeronautics Program.

\section{References}

${ }^{1}$ Watson, W. R., Nark, D. M., and Jones, M. G., "Assessment of 3-D Codes for Predicting Liner Attenuation in Flow Ducts," AIAA Paper 2008-2828, 2008.

${ }^{2}$ Dougherty, R. P., “A Wave-Splitting Technique for Nacelle Acoustic Propagation,” AIAA Paper 97-1652, 1997.

${ }^{3}$ Dougherty, R. P., "A Parabolic Approximation for Flow Effects on Sound Propagation in Nonuniform, Softwall, Ducts," AIAA Paper 99$1822,1999$.

${ }^{4}$ Nark, D. M., Envia, E., and Burley, C. L., "Fan Noise Prediction with Applications to Aircraft System Noise Assessment," AIAA Paper 2009-3291, 2009.

${ }^{5}$ Nark, D. M., Envia, E., and Burley, C. L., “On Acoustic Source Specification for Rotor-Stator Interaction Noise Prediction,” AIAA Paper 2010-3713, 2010.

${ }^{6}$ Weir, D., "Engine Validation of Noise and Emission Reduction Technology Phase I," NASA CR 2008-215225, 2008.

${ }^{7}$ Nark, D. M., Farassat, F., Pope, D. S., and Vatsa, V., "The Development of the Ducted Fan Noise Propagation and Radiation Code CDUCTLaRC," AIAA Paper 2003-3242, 2003.

${ }^{8}$ Lan, J. H., "Turbofan Duct Propagation Model,” NASA CR 2001-211245, 2001.

${ }^{9}$ Brentner, K. B. and Farassat, F., "Analytical Comparison of the Acoustic Analogy and Kirchhoff Formulation for Moving Surfaces," AIAA Journal, Vol. 36, No. 8, 1998, pp. 1379-1386.

${ }^{10}$ Schuster, B., Lieber, L., and Vavalle, A., "Optimziation of a Seamless Inlet Liner Using an Empirically Validated Prediction Method," AIAA Paper 2010-3824, 2010.

${ }^{11}$ Zlavog, G. and Eversman, W., "Source effects on attenuation in lined ducts. Part I: A statistically based computational approach," Journal of Sound and Vibration, Vol. 307, No. 1-2, 2007, pp. 113-138.

${ }^{12}$ Zlavog, G. and Eversman, W., "Source effects on attenuation in lined ducts. Part II: Statistical properties," Journal of Sound and Vibration, Vol. 307, No. 1-2, 2007, pp. 139-151.

${ }^{13}$ Coleman, H. W. and Steele, W. G., Experimentation and Uncertainty Analysis for Engineers, John Wiley \& Sons, Inc., 1999.

${ }^{14}$ Envia, E., Tweed, D. L., Wooward, R. P., Elliott, D. M., Fite, E. B., Hughes, C. E., Podboy, G. G., and Sutliff, D. L., "An Assessment of Current Fan Noise Prediction Capability," AIAA Paper 2008-2991, 2008. 


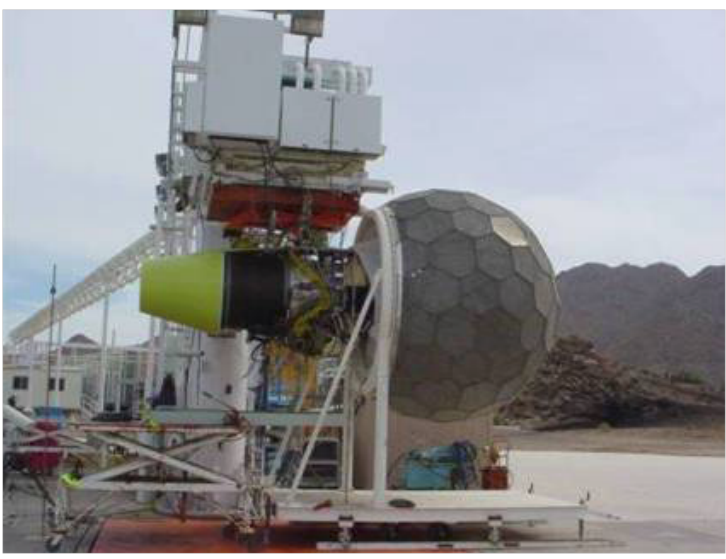

(a) Tech977 turbofan engine mounted on test rig with ICD

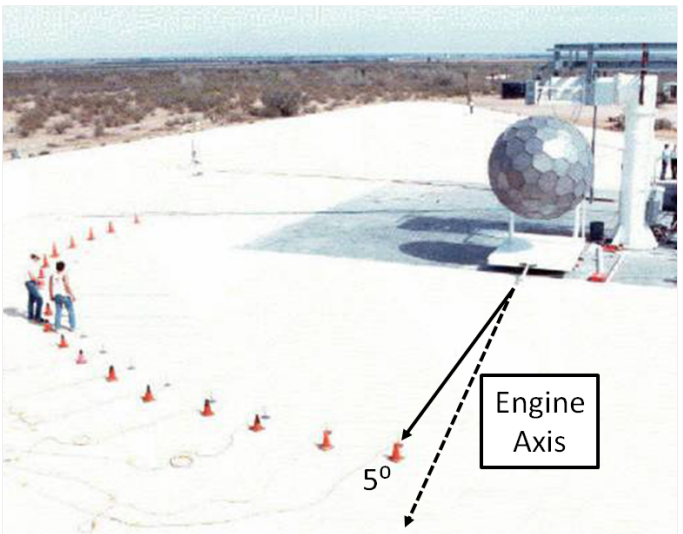

(b) Far-field microphone configuration

Figure 1: TECH977 turbofan engine inlet test configuration. ${ }^{6}$

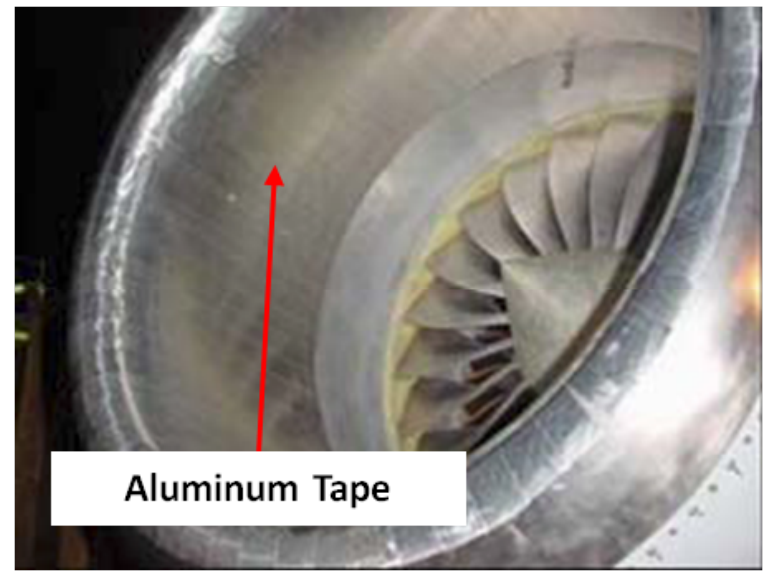

(a) Hardwall

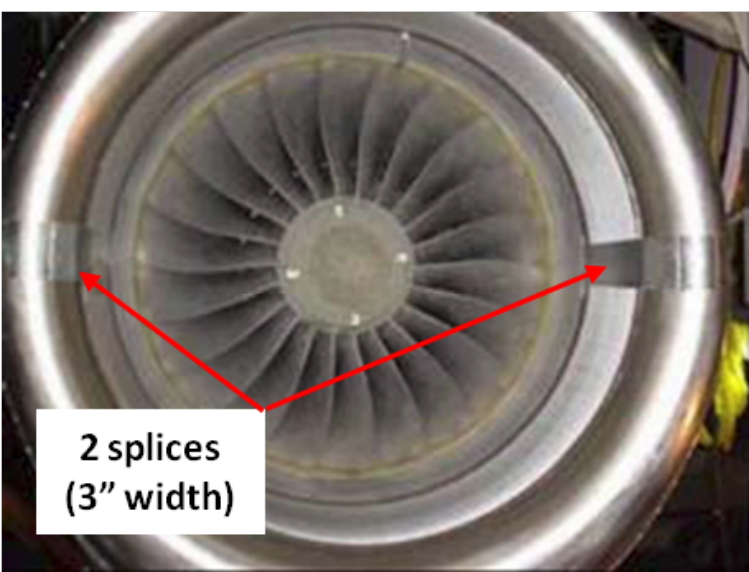

(c) Two Splices of three-inch width

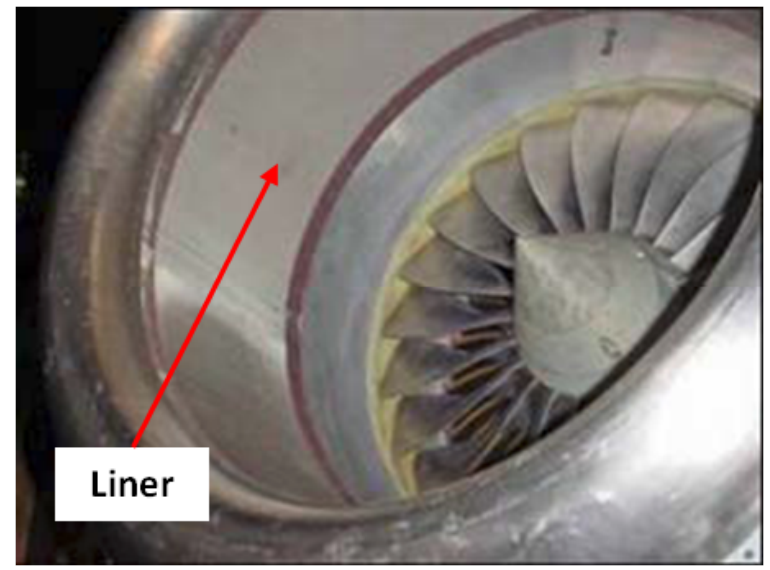

(b) Seamless Liner

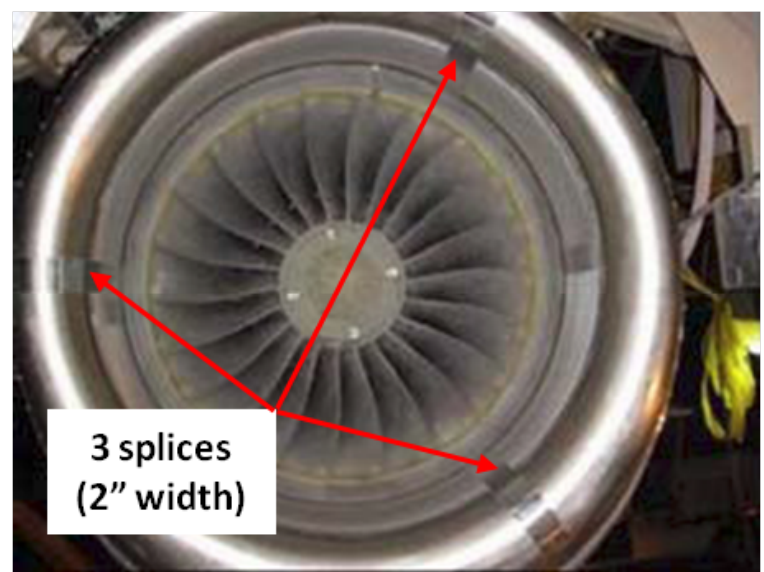

(d) Three Splices of two-inch width

Figure 2: Liner Splice Configurations for Far Field Inlet Measurements ${ }^{6}$ 


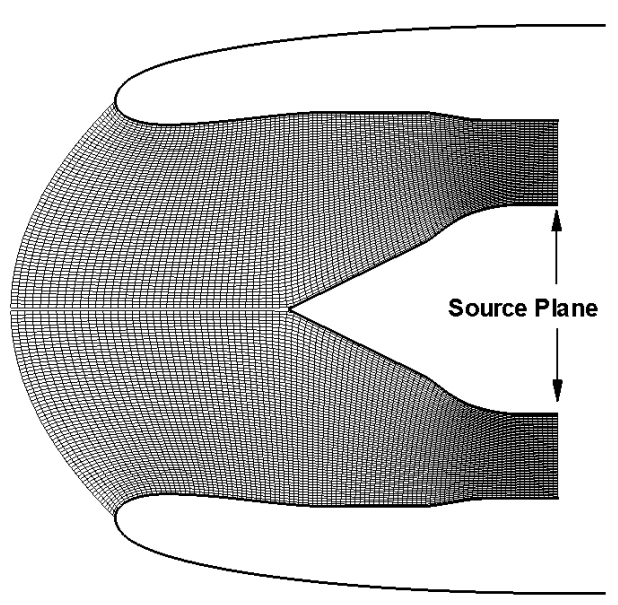

(a) Internal duct propagation grid

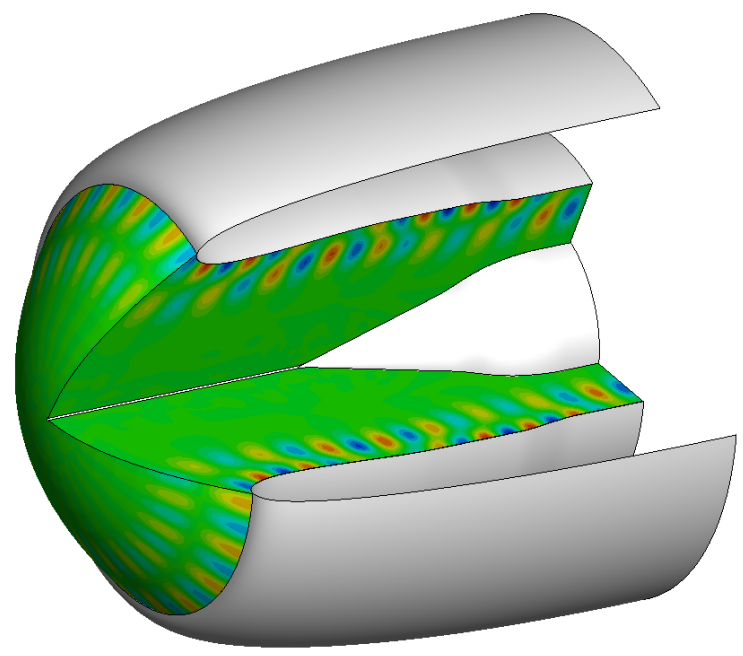

(b) Example internal duct propagation prediction

Figure 3: Example CDUCT-LaRC Internal Inlet Fan Noise Predictions.

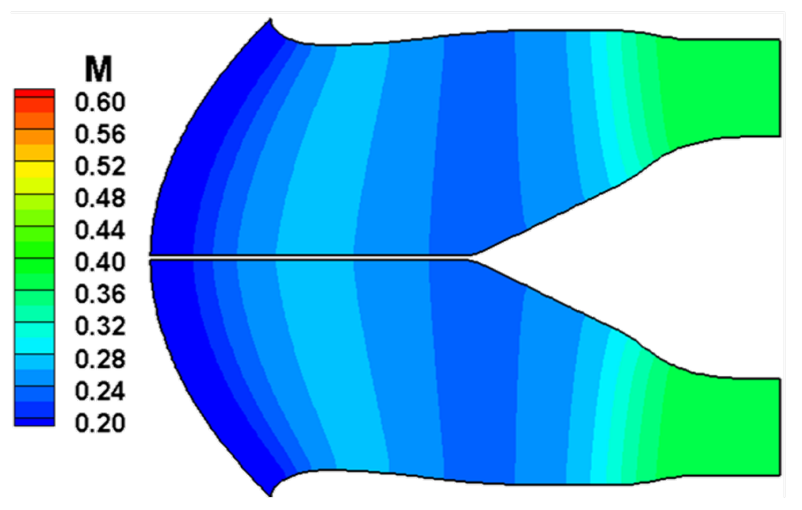

(a) $54 \% \mathrm{~N} 1 \mathrm{C}$

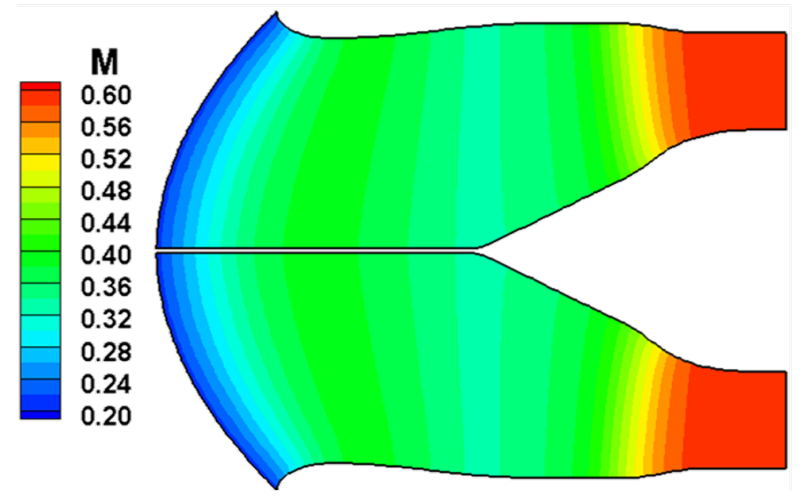

(b) $87 \% \mathrm{~N} 1 \mathrm{C}$

Figure 4: Mean flow Mach number contours for internal duct propagation predictions. 


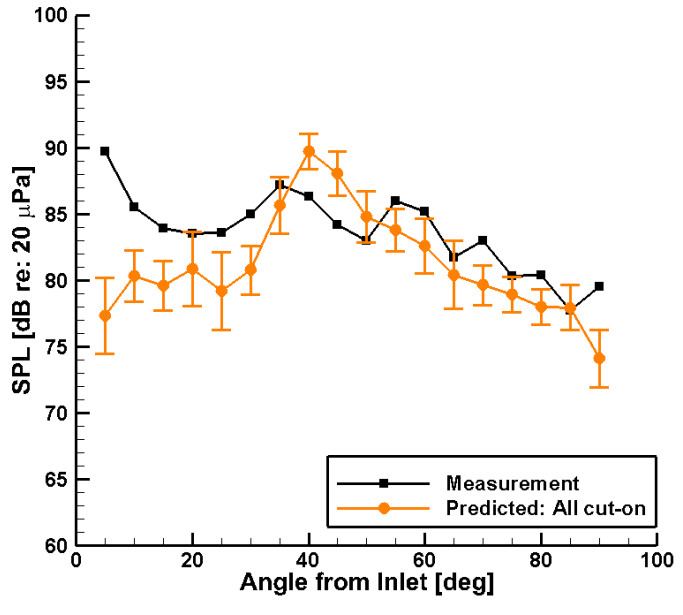

(a) Directivity pattern for hardwall configuration.

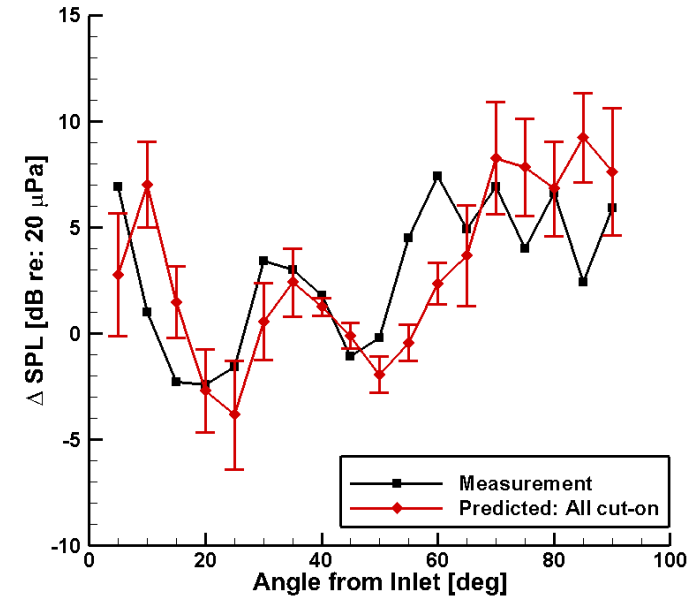

(b) Delta SPL between hardwall and seamless liner.

Figure 5: BPF predictions using all cut-on modes and measured data at 54\% power. Error bars denote the $95 \%$ confidence interval for the predicted quantities.

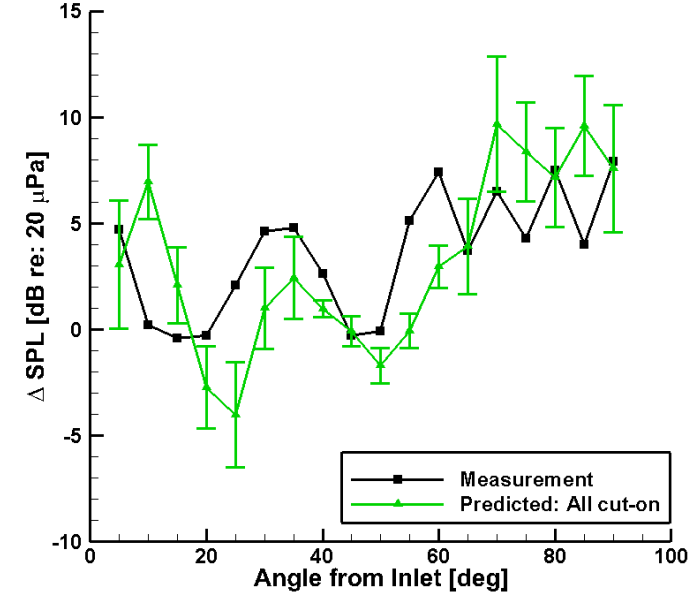

(a) Delta SPL between hardwall and 2-splice liner configuration.

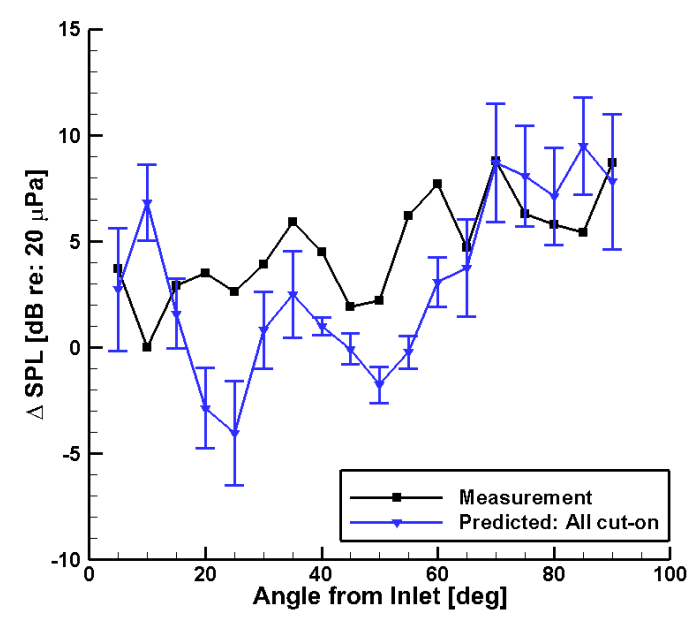

(b) Delta SPL between hardwall and 3-splice liner configuration.

Figure 6: BPF predictions using all cut-on modes and measured data at 54\% power. Error bars denote the $95 \%$ confidence interval for the predicted quantities. 


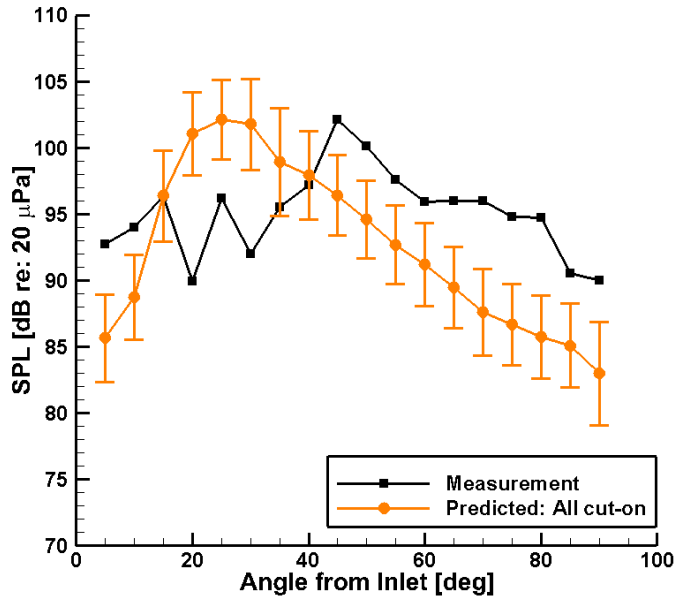

(a) Directivity pattern for hardwall configuration

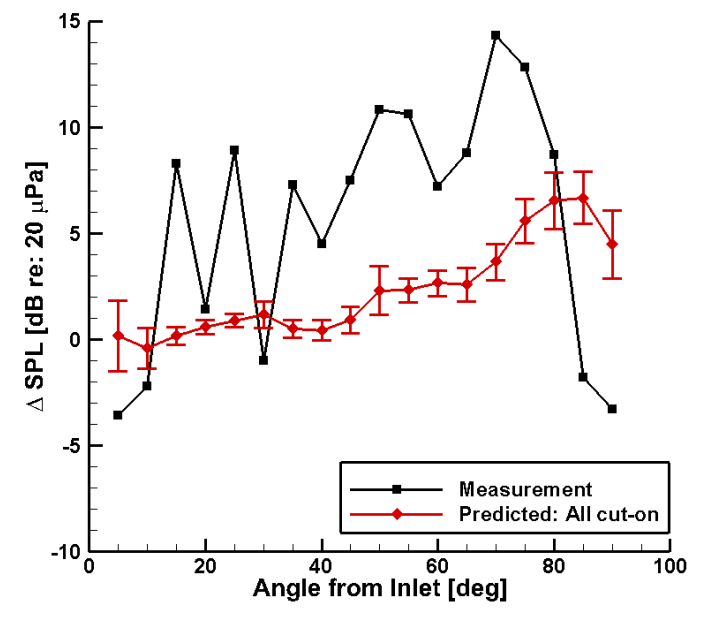

(b) Delta SPL between hardwall and seamless liner.

Figure 7: BPF predictions using all cut-on modes and measured data at $87 \%$ power. Error bars denote the $95 \%$ confidence interval for the predicted quantities.

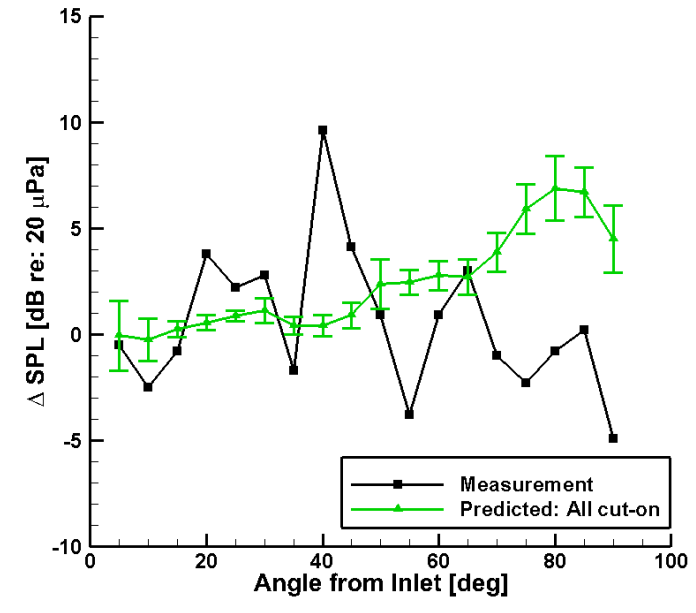

(a) Delta SPL between hardwall and 2-splice liner configuration.

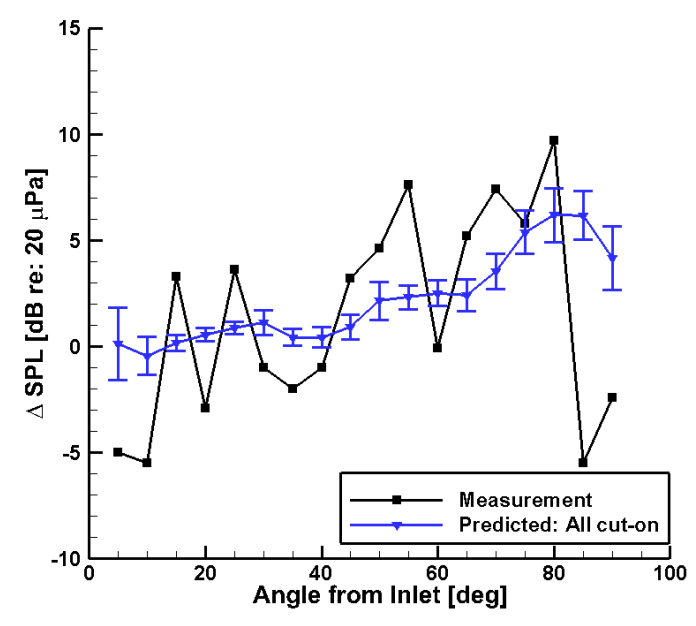

(b) Delta SPL between hardwall and 3-splice liner configuration.

Figure 8: BPF predictions using all cut-on modes and measured data at $87 \%$ power. Error bars denote the $95 \%$ confidence interval for the predicted quantities. 


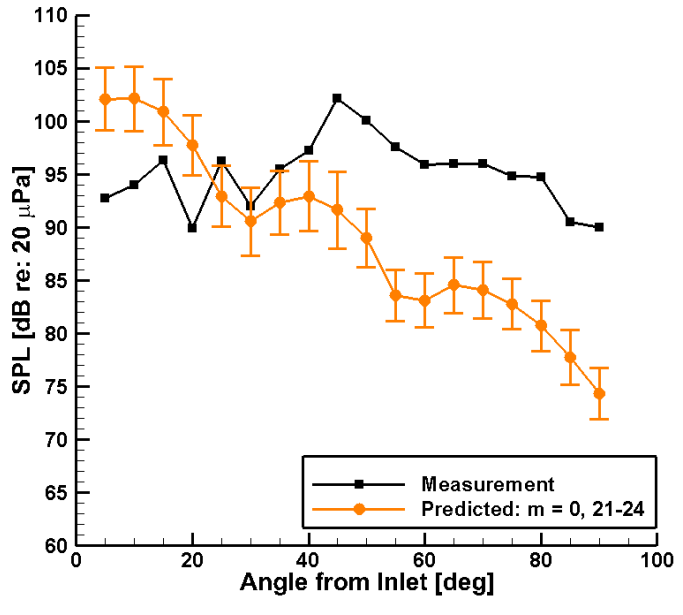

(a) Directivity pattern for hardwall configuration

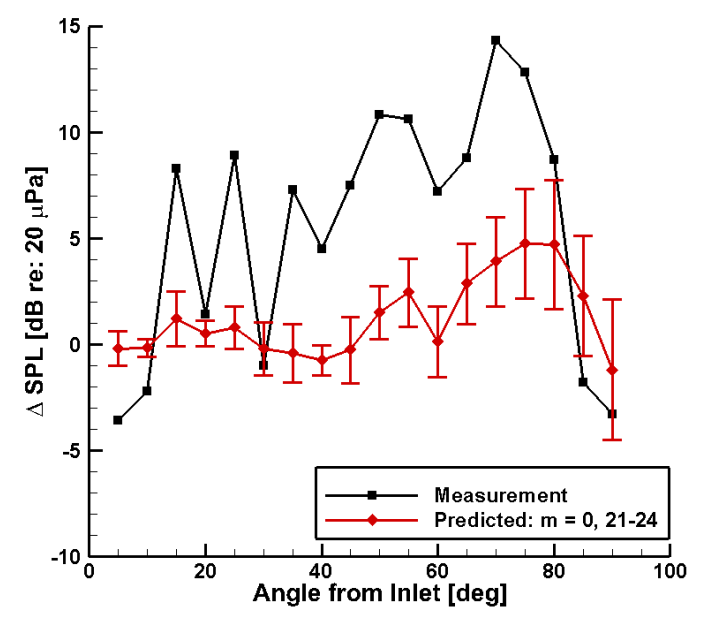

(b) Delta SPL between hardwall and seamless liner.

Figure 9: BPF predictions using $m=0,21-25$ circumferential modes (all cut-on radial modes) and measured data at $87 \%$ power. Error bars denote the $95 \%$ confidence interval for the predicted quantities.

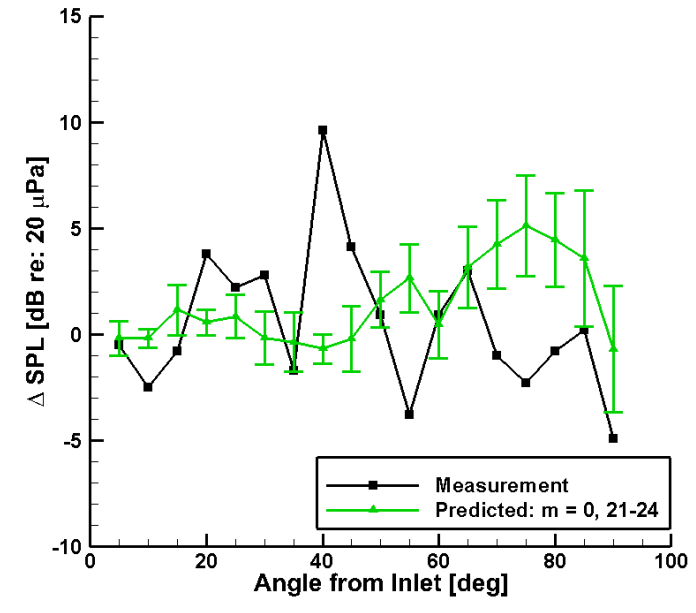

(a) Delta SPL between hardwall and 2-splice liner configuration.

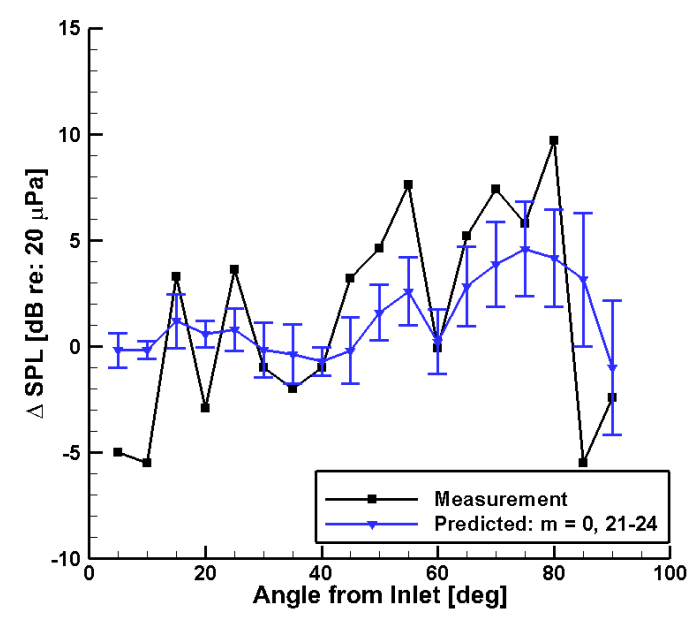

(b) Delta SPL between hardwall and 3-splice liner configuration.

Figure 10: BPF predictions using $m=0,21-25$ circumferential modes (all cut-on radial modes) and measured data at $87 \%$ power. Error bars denote the $95 \%$ confidence interval for the predicted quantities. 